
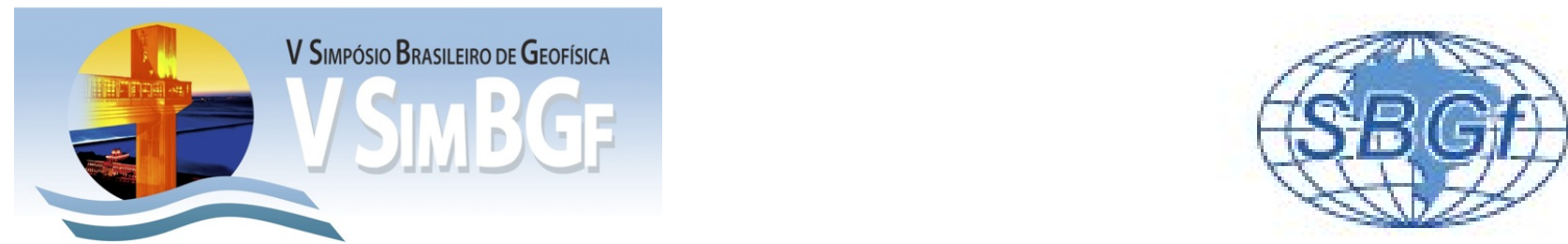

\title{
Caracterização do Reservatório Marlim por Inversão Acústica
}

Tatiane M. Nascimento FGEL/UERJ-Invision Geophysics, Paulo T. L. Menezes-DGAP/FGEL/UERJ-PETROBRAS, Igor L. Braga-Invision Geophysics

Copyright 2012, SBGf - Sociedade Brasileira de Geofísica Este texto foi preparado para a apresentação no $V$ Simpósio Brasileiro de Geofísica, Salvador, 27 a 29 de novembro de 2012. Seu conteúdo foi revisado pelo Comitê Técnico do $V$ SimBGf, mas não necessariamente representa a opinião da SBGf ou de seus associados. É proibida a reprodução total ou parcial deste material para propósitos comerciais sem prévia autorização da SBGf.

\section{RESUMO}

No presente trabalho apresentamos os resultados preliminares do estudo de inversão acústica, aplicada aos dados sísmicos 3D, no Campo de Marlim, Bacia de Campos. O trabalho foi desenvolvido em um workflow dividido em três principais etapas: condicionamento do dado sísmico; amarração dos poços; e por último, inversão/interpretação.

O cubo de impedância acústica gerando possui mais alta resolução que o dado sísmico em amplitude, permitindo uma melhor visualização das feições geológicas dos turbiditos de Marlim bem como uma estimativa de porosidade para estes.

\section{INTRODUÇÃO}

Para o complexo processo de caracterização de reservatório, é necessário estar de posse de uma série de informações. Estas, quando integradas, permitem traçar estratégias de avaliação de um campo, bem como seu posterior monitoramento. Nesse sentido, as informações obtidas através de dados sísmicos 3D são de grande relevância na exatidão do modelo geológico a ser gerado (Martins et al., 1995).

Isto é válido para reservatórios turbidíticos de águas profundas. Uma vez que a grande complexidade de distribuição dos corpos de areia e de suas espessuras associadas, por muitas vezes são menores que o limite de resolução vertical do dado sísmico (Ribeiro, 2012). Surge ai, como forma de quantificar os diversos parâmetros petrofísicos de um reservatório de forma mais realista possível, a necessidade de junção de dados diversos e com diferentes graus de precisão. Destacam-se: perfis de poços, dados sísmicos 3D e modelos geológicos conceituais. A recuperação desses parâmetros petrofísicos, através da inversão do dado sísmico para im- pedância acústica, tem demonstrado ser uma excelente ferramenta de união dessas informações para criação de modelos mais precisos (Sancevero, 2006).

Além disso, o resultado da inversão permite a extração de informações geológicas relativas à litologia e ao fluido, e não somente à variação de interface, como o obtido através do dado em amplitude (Avseth et al. 2005). Sendo a impedância acústica uma propriedade relativa à camada, e não à interface, o processo de inversão adiciona informação ao dado sísmico, possibilitando uma maior inferência da geologia de subsuperfície (Bacon et al., 2003).

No presente trabalho apresentamos resultados preliminares da aplicação de inversão acústica a dados sísmicos 3D do campo de Marlim (Bacia de Campos). Utilizamos 4 poços disponíveis no campo de modo a calibrar todo o processo e obter um modelo de baixa frequência estratigraficamente coerente. Este processo permitiu uma melhor interpretação dos parâmetros geológicos do complexo turbidítico de marlim.

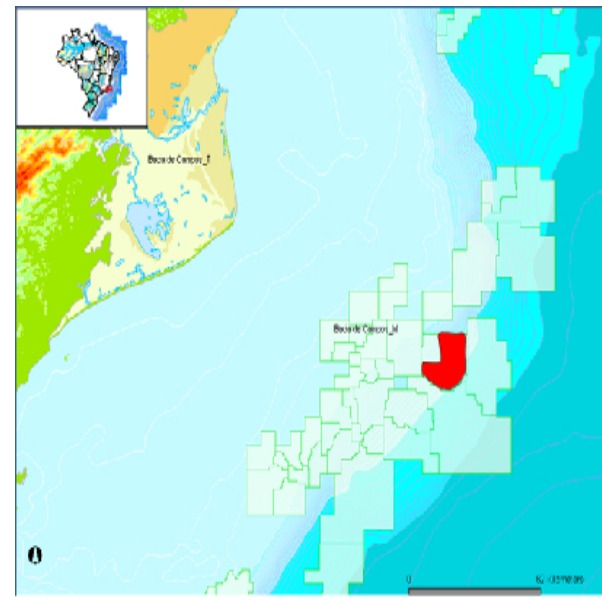

Figura 1: Localizaçao do Campo de Marlim, Bacia de Campos.

\section{ÁREA DE ESTUDO E DADOS UTILIZADOS}

O estudo foi realizado no Campo de Marlim, localizado na porcão noroeste da Bacia de Campos, a aproximadamente a $110 \mathrm{~km}$ de distancia da costa (Figura 1). O reservatório do Campo de Marlim é constituído por um sistema turbidítico de águas profundas, depositado 
durante o Oligoceno, compondo uma série de corpos arenosos amalgamados, resultando em um único intervalo produtor (Peres, 1993). A trapa do reservatório possui fechamento estratigráfico a oeste, norte e sul, dada pelo pinch out das camadas de areia, e para leste, a acumulação tem seu limite contra uma falha normal lístrica (Candido e Cora, 1978).

De acordo com Sansonowski et al. (2007), a porosidade do reservatório do Campo de Marlim se encontra em torno de 30 porcento. A resposta sísmica sobre o campo revela eventos de alta amplitude (característica comum em complexos turbiditicos não confinados), resultado do contraste desta com as zonas de alta porosidade no reservatório, quando comparadas com a relativa baixa velocidade do folhelho sobrejacente (Fainstein et al., 2001).

Os dados utilizados no estudo foram um cubo sísmico 3D pós-stack e curvas de perfis de quatro poços, adquiridos junto à Agência Nacional de Petróleo (ANP). Estas seções possuem profundidade máxima de penetração do sinal sísmico de 5 segundos em tempo duplo de reflexão a partir do nível do mar.

Foi estabelecido um workflow de trabalho em três etapas principais:

Primeira etapa: filtragem dos dados sísmicos. Tratou-se do condicionamento do dado sísmico visando obter um cubo sísmico com mais alta resolução e continuidade dos traços de amplitude. Para tal foi aplicado um filtro para atenuação de ruídos aleatórios, usando a transformada de curvlet.

Segunda etapa: amarração com poços. Com o intuito de correlacionar os topos estratigráficos interpretados nos poços, com os horizontes em tempo na sísmica, foram utilizados os perfis de densidade e sônico. A partir desses dois foi criada uma curva de impedância acústica para cada poço e também se extraiu a wavelet para cada poço. Geração de sismograma sintético.

Terceira etapa: inversão determinística do dado sísmico, utilizando a metodologia tipo model based e interpretação dos resultados. Geração de um modelo de baixa frequência consistente com a estratigrafia local, com a criação de um cubo contendo os valores de direção e mergulho, extraídos do dado sísmico. Em seguida, quatro horizontes sísmicos principais foram interpretados no dado em amplitude, e em conjunto com as informações prévias de direção e mergulho, as sequências deposicionais intermediárias a estes horizontes foram extrapoladas. Para compor o espectro sísmico de baixa frequência do dado, foram filtradas dos poços, as curvas de impedância nas frequências de 0 a $10 \mathrm{~Hz}$. Estas curvas foram interpoladas com o cubo contendo os horizontes extrapolados, resultando no modelo de baixa frequência. Finalmente, o modelo de baixa frequência foi unido ao resultado da inversão do dado sísmico 3D, originando ao volume final da inversão.

\section{RESULTADOS}

O volume final da inversão sísmica possui imageamento superior quando comparado ao dado original (figura 2a). Esta melhoria, dentre outros aspectos, está atrelada à remoção do efeito da wavelet, inerente ao processo de inversão. A transformação da propriedade de interface do dado sísmico em propriedade de camada, possibilitando maior delimitação visual do corpo investigado, compõe uma das razões que fazem da inversão uma ferramenta mais precisa para a caracterização de reservatório. As feições deposicionais do corpo turbidítico, que forma o reservatório do Campo de Marlim, foram meIhor delineadas após a inversão do dado sísmico (figura $2 b)$.

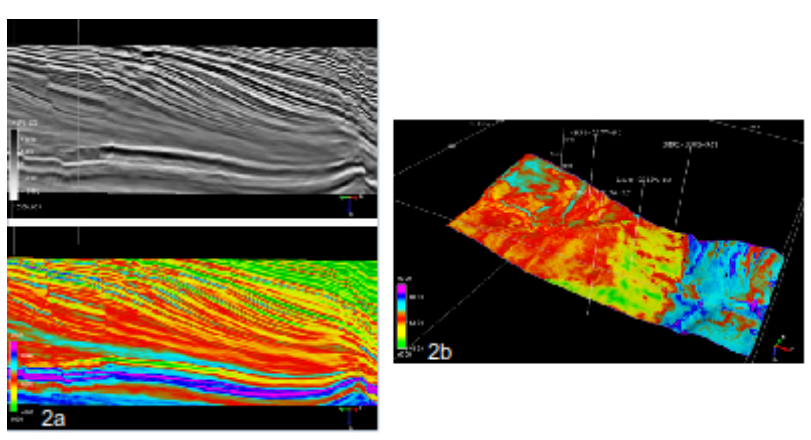

Figura 2: a) Painel superior - in-line do dado em amplitude; painel inferior - in-line do dado IP abs. b) Painel direito - Horizonte do turbidito Marlim - impedância acústica.

Ilustramos no painel inferior da figura 3 a comparação entre a curva curva de impedância do poço poço 1-RJ219-A (filtrada na banda sísmica do dado original), com a curva de impedância absoluta (IP abs), oriunda da inversão. Observa-se nesta figura uma boa correlação entre as impedâncias obtidas no poço e no dado sísmico.

Como dito anteriormente, os dados de poço são importantes fontes de informação, que correlacionadas com o dado sísmico diminuem as incertezas a respeito do alvo investigado. Dados como curvas de poço, testes de pressão e de propriedades elásticas, são alguns dos muitos dados que auxiliam na calibração dos poços, através das correlações empíricas entre estes (Wagner et al., 2012).

Neste estudo, as curvas de porosidade $(\varphi)$ foram usadas para estabelecer relações entre os valores encontrados no poço e o resultado da impedância acústica derivada da inversão (IP abs). Para auxiliar na identificação litológica, também foram utilizados os dados de raios gama. Estes valores quando atribuídos ao gráfico de correlação de IP abs $\times \varphi$, possibilitam a delimitação de regiões correspondentes as areias do reservatório. Evidentemente, 


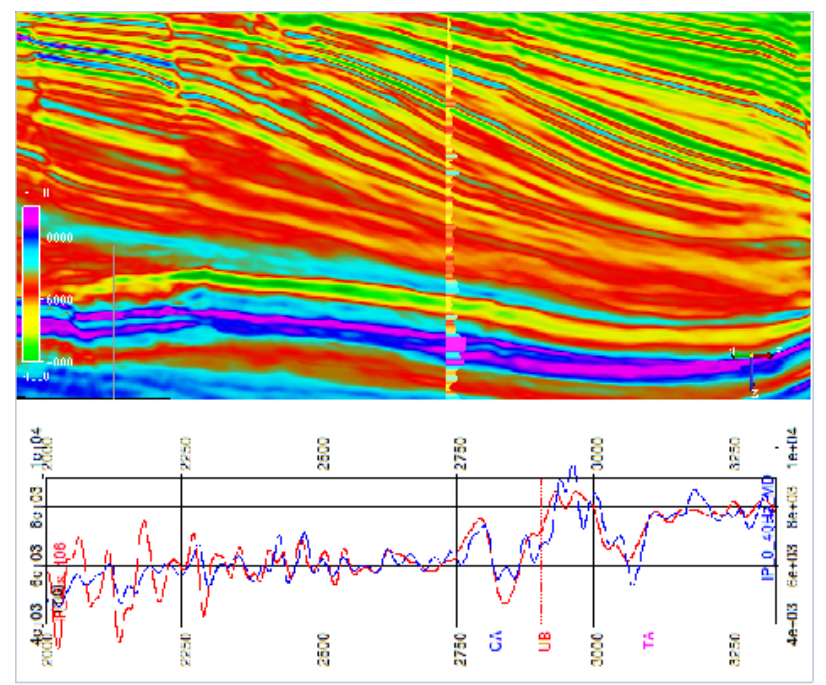

Figura 3: Comparação resultados poço 1-RJ-219A com IP abs obtido pela inversão. Painel superior - comparação na in-line sísmica. Painel inferior comparação em um traço.

essa ferramenta possui limitações, pois a complexidade mineralógica associada as interlaminações de camadas de follhelho pouco espessas, presentes no reservatório, necessita de caraterizações mais acuradas, como as realizadas através do estudo de física de rochas.

Conforme observado na figura $2 b$, as impedâncias mais baixas correspondem às fácies dos turbiditos Marlim. Quando separamos a nuvem de pontos, referentes a tais valores de impedância no gráfico IP abs $\times \varphi$, notamos que estes traduzem porosidades encontradas entre os eixos correspondentes a 30 e 40 porcento, acordando com o encontrado na literatura. Na figura 4, os pontos selecionados no gráfico IP abs $\times \varphi$ foram plotados na imagem sísmica, onde é possível notar que estes se concentram na região do reservatório.

\section{DISCUSSÃO E CONCLUSÕES}

Os resultados obtidos no presente estudo demonstram a importância do papel da inversão sísmica no auxilio da caracterização de reservatórios, principalmente quando se trata da delimitação de corpos apresentando afinamento estratigráfico, como é o caso de reservatórios turbidíticos de Marlim.

Um imageamento consistente com a geologia em estudo é essencial, tanto para a delimitação regional das superfícies de interesse, quanto para a modelagem em escala de reservatório. Dessa forma, os resultados da inversão sísmica podem ser usados amplamente, desde a fase de exploração ate o desenvolvimento do campo. Integrando as informações advindas dos poços com o resultado da inversão sísmica, potencializa-se esta ferramenta, podendo ser gerados modelos geológicos mais precisos e por assim otimizar as previsões acerca do reservatório.

\section{AGRADECIMENTOS}

Agradecemos a DGB Earth Sciences a disponibilização sem custos do software de interpretação sísmica Opendtect (www.opendtect.org) e a Invision Geophysics a utilização de seu parque comptacional para a realização desta pesquisa. PTLM agradece ao suporte fornecido por bolsa de pesquisa do CNPq. Agradecemos também ao suporte adicional fornecido pelo projeto CNPq no.470742/2011-9.

\section{REFERÊNCIAS}

Bacon, M., Simm, R., e Redshaw, T., 2003, 3-d seismic interpretation: Cambridge Univ $\mathrm{Pr}$.

Candido, A., e Cora, C., 1978, The marlim and albacora giant fields, campos basin, offshore brazil: AAPG Memoir, 54, 123-135.

Fainstein, R., Jamieson, G., Hannan, A., Eiles, N., Krueger, A., e Schelander, D., 2001, Offshore brazil santos basin exploration potential from recently acquired seismic data: Offshore brazil santos basin exploration potential from recently acquired seismic data:, 7th International Congress of the Brazilian Geophysical Society, 52-55.

Martins, C. C., Da Costa, C. A., Theodoro, C. E., Guardado, L. R., e Andrade, V. F., 1995, 3-d seismic; a successful strategy in the campos basin: The Leading Edge, 14, no. 6, 701-704.

Peres, W. E., 1993, Shelf-fed turbidite system model and its application to the oligocene deposits of the campos basin, brazil: AAPG BULLETIN, 77, 81-81.

Ribeiro, N., 2012, Joint inversion of multi-component seismic data for reservoir characterization of an offshore campos basil field, brazil: First Break, 30, no. 5, 87-91.

Sansonowski, R., de Oliveira, R., Júnior, N., Bampi, D., e amarão Junior, L., 2007, 4d seismic interpretation in the marlim field, campos basin, offshore brazil: $4 \mathrm{~d}$ seismic interpretation in the marlim field, campos basin, offshore brazil:, 2007 SEG Annual Meeting.

Wagner, C., Gonzalez, A., Agarwal, V., Koesoemadinata, A., Ng, D., Trares, S., Biles, N., e Fisher, K., 2012, Quantitative application of poststack acoustic impedance inversion to subsalt reservoir development: The Leading Edge, 31, no. 5, 528-537. 


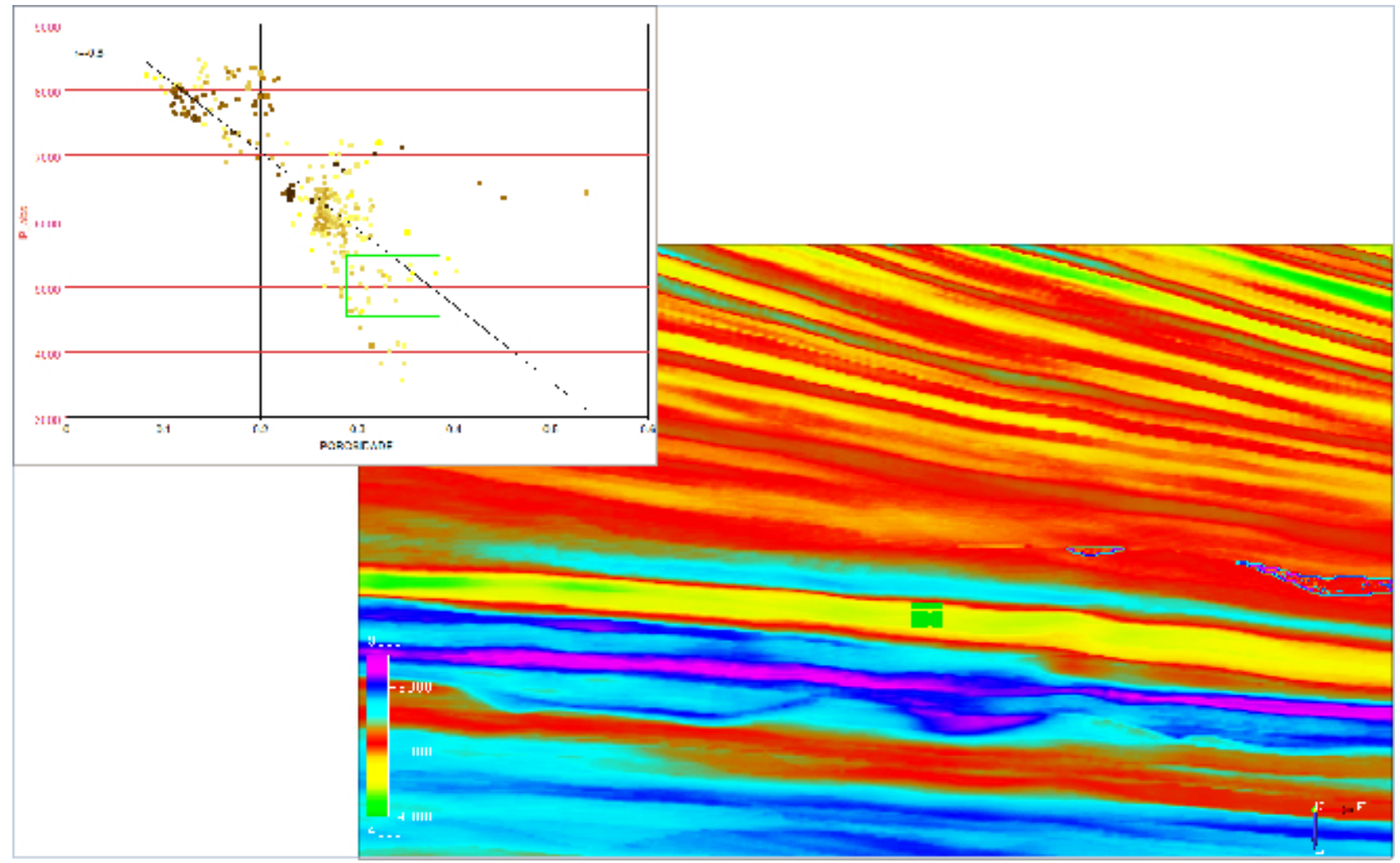

Figura 4: Painel superior esquerdo - Gráfico IP abs $x \varphi$. Painel inferior direito in-line de IP abs. Os valores de porosidade dentro da área verde demarcada no gráfico foram plotados na imagem sísmica, correspondendo às fácies reservatório. 